Check for updates

Cite this: RSC Adv., 2019, 9, 28291

Received 5th August 2019

Accepted 26th August 2019

DOI: $10.1039 / c 9 r a 06069 c$

rsc.li/rsc-advances

\title{
Enhancement of efficiency and CCT uniformity for red phosphor thin films, red LEDs and laminated white LEDs based on near-ultraviolet LEDs using MgO nanoparticles
}

\begin{abstract}
Ningze Zhuo, (D) abc Na Zhang, ${ }^{\text {bc }}$ Peng Chen ${ }^{\star a}$ and Haibo Wang*bc
Red phosphor thin films (PTFs) with different MgO nanoparticle concentrations for near-ultraviolet (NUV) LEDs were prepared based on their strong scattering effect; red LEDs and laminated white LEDs were packaged further. SEM and XRD showed that $\mathrm{CaAlSiN}_{3}: \mathrm{Eu}^{2+}(\mathrm{CASN})$ and $\mathrm{MgO}$ nanoparticles were uniformly distributed in silicone resin and their crystal structure remained unchanged. The phosphor conversion efficiency (PCE) had a maximum value of $83.15 \%$ when the $\mathrm{MgO}$ nanoparticle concentration was 15 wt\%. An increase of concentration can improve the spatial distribution uniformity of photonics for $410 \mathrm{~nm}, 627 \mathrm{~nm}$ and $660 \mathrm{~nm}$. Fluorescence lifetime showed that the value is positively correlated with concentration change. The packaged red LED luminous flux reaches a maximum of $20.337 \mathrm{~lm}$ at a concentration of $15 \mathrm{wt} \%$. The laminated white LED showed that the $\mathrm{MgO}$ nanoparticle concentration can be used to adjust the correlated color temperature (CCT) from $4322 \mathrm{~K}$ to $1987 \mathrm{~K}$. Under similar CCT, the red phosphor concentration is only $1.83 \mathrm{wt} \%$, the dosage is reduced by $56.12 \%$, and the corresponding luminous efficiency of radiation (LER) and luminous efficiency (LE) are $296.03 \mathrm{~lm} \mathrm{~W}^{-1}$ and $73.72 \mathrm{Im} \mathrm{W}^{-1}$ respectively. The increase was $11.42 \%$, the decrease was $10.14 \%$, the color rendering index (CRI) increased from 90.6 to 91.8 , and CCT uniformity increased from $82.04 \%$ to $89.27 \%$ with an increase of $8.81 \%$. Research shows that $\mathrm{MgO}$ nanoparticles have potential application value in the preparation of high-quality white LEDs.
\end{abstract}

\section{Introduction}

Over the past decade, white LEDs have achieved rapid development owing to their high luminous efficiency, being spectrally adjustable, fast response, environmental friendliness, etc. ${ }^{1,2}$ Nowadays, the main way to realize white LEDs is that a blue LED chip excites a yellow $\mathrm{Y}_{3} \mathrm{Al}_{5} \mathrm{O}_{12}: \mathrm{Ce}^{3+}$ phosphor; due to the lack of red spectrum, the light shows high CCT and low CRI. However, high-quality white LEDs with full-spectrum and highCRI characteristics are gradually attracting general interest nowadays. NUV LED chips exciting multi-primary phosphors is another way to achieve high-quality white LEDs. This has the advantages of wide spectral distribution, high CRI, and stable light color performance. ${ }^{3,4}$ Studies have shown that owing to differences of field environment of phosphor matrix crystals, there is an overlap between the absorption and emission spectra of different phosphors, that is, the spectral re-

${ }^{a}$ School of Electronic Science and Engineering, Nanjing University, Nanjing 210093, China.E-mail: pchen@nju.edu.cn

${ }^{b}$ Institute of Optoelectronic Materials of Light Industry, Nanjing 210015, China. E-mail:wanghaibo88@163.com

${ }^{c}$ Research Institute of Electric Light Source Materials, Nanjing Tech University, Nanjing 210015, China absorption effect..$^{5-7}$ Therefore, the traditional hybrid coating structure easily causes the mutual absorption of energy and the internal energy consumption of photons of different wavelengths. In response to this phenomenon, researchers used stratification or partition of PTFs to attenuate the re-absorption and improve the device performances..$^{8-11}$

As phosphor conversion materials, ${ }^{12-14}$ PTFs can convert the absorbed photons into an emission spectrum with specific energy distribution. With wide application range, in the display field, ${ }^{\mathbf{1 5 , 1 6}}$ PTFs can effectively improve color uniformity and light extraction efficiency of fluorescent screens; in the field of photovoltaics, ${ }^{17,18}$ they can effectively improve utilization efficiency of solar energy; in the field of solid-state lighting, ${ }^{19,20}$ also known as remote phosphors, they have the advantages of improving uniformity and photo-thermal stability. ${ }^{21-23}$ Researchers have used a variety of methods to further optimize the performance of PTF-based white LEDs, such as surface plasmons ${ }^{24}$ gradient index design, ${ }^{25}$ graphical processing ${ }^{26-29}$ free-form surface design, ${ }^{30}$ polarization effect ${ }^{31}$ and so on. Kwon $^{24}$ enhanced luminescence properties by combining a waveguiding ZnO-based nanostructure with plasmonic $\mathrm{Au}$ nanoparticles of PTFs, and improved the LE of white LEDs by $18 \% . \mathrm{Xu}^{25}$ prepared phosphor in glass with gradient refractive 
index structure by screen printing, breaking the total reflection effect in the process of photon propagation, and the LE was improved by $7.81 \%$. $\mathrm{Kim}^{26}$ used the nano-texture technology of glass substrate to obtain PTFs with patterned structure, improving the efficiency by $16 \%$, and the simulated result showed that a large amount of light was scattered from the patterned structure. $\mathrm{Li}^{\mathbf{3 0}}$ improved the uniformity of PTFs by $26.2 \%$ through the design of a double free-form surface. In addition to the above methods, nanoparticles have a great advantage in improving white LED light color performance due to their strong scattering effect. ${ }^{32-36}$ Tang $^{32}$ introduced inorganic ZnO scattering particles through quantum dots, the dispensing method achieving a 3.37\% improvement in QD-LED efficiency. The CCT difference from $862 \mathrm{~K}$ to $712 \mathrm{~K}$ is reduced from $-70^{\circ}$ to $70^{\circ}$. Chen ${ }^{33}$ reported the use of $\mathrm{ZrO}_{2}$ nanoparticles; the luminous flux is increased by $12 \%$ at a concentration of $1 \mathrm{wt} \%$ and the CCT difference is reduced from $522 \mathrm{~K}$ to $7 \mathrm{~K}$ when the concentration is increased to $10 \%$.

The aforementioned studies are mostly limited to blue LED chips, and a single type of PTF or phosphor. Different from the above research, in this paper, red PTFs with MgO nanoparticles for laminated NUV white LEDs are used as the research object, systematically exploring the regularity of microstructure, reflectivity and transmittance, PCE, fluorescence lifetime of PTFs, LE and CCT uniformity of packaged red LED and laminated white light LED. It is found that the scattering effect of $\mathrm{MgO}$ nanoparticles can effectively improve the PCEs of PTFs and luminous flux of red LEDs. In the application of laminated white LEDs, the CCT regulation can be controlled by the adjustment of $\mathrm{MgO}$ nanoparticle concentration; the incorporation of $\mathrm{MgO}$ nanoparticles can effectively improve the CCT uniformity, and save a large amount of red phosphor, which has the obvious advantage of reducing the production cost.

\section{Experimental methods}

\section{Experimental materials}

PTF-based red LED and laminated white LED structures are shown in Fig. 1. The chemical compositions of blue, green and red phosphors are $(\mathrm{Ba}, \mathrm{Sr})_{10}\left(\mathrm{PO}_{4}\right)_{6} \mathrm{Cl}_{2}: \mathrm{Eu}^{2+},(\mathrm{Ba}, \mathrm{Sr})_{2} \mathrm{SiO}_{4}: \mathrm{Eu}^{2+}$, and $\mathrm{CaAlSiN}_{3}: \mathrm{Eu}^{2+}$, with corresponding emission peaks of $445 \mathrm{~nm}, 520 \mathrm{~nm}$ and $627 \mathrm{~nm}$. For MgO nanoparticles with $D_{50}=$ $0.61 \mu \mathrm{m}$, refractive index is $1.70 ; \mathrm{CaAlSiN}_{3}: \mathrm{Eu}^{2+}$ with $D_{50}=18.7$ $\mu \mathrm{m}$, refractive index is 2.19. The silicone resin is Dow Corning MS-1002 (composed of A and B dual components), with refractive index of 1.41 . The NUV LED chip size is $14 \mathrm{mil} \times 28$ mil, the peak wavelength is $415 \mathrm{~nm}$, and the SMD lead frame size is $5.6 \mathrm{~mm} \times 3 \mathrm{~mm} \times 0.7 \mathrm{~mm}$. The blue phosphor concentration is $100 \mathrm{wt} \%$ (A : B : blue phosphor $=1.50 \mathrm{~g}: 1.50$ $\mathrm{g}: 3.00 \mathrm{~g})$, the green phosphor concentration is $24.17 \mathrm{wt} \%$ (A : B : green phosphor $=1.50 \mathrm{~g}: 1.50 \mathrm{~g}: 0.725 \mathrm{~g}$ ), and the red phosphor concentrations are $1.83 \mathrm{wt} \%, 2.33 \mathrm{wt} \%, 2.83 \mathrm{wt} \%$, 3.33 wt $\%, 3.83$ wt $\%, 4.17$ wt $\%$ (A : B : red phosphor $=1.50$ $\mathrm{g}: 1.50 \mathrm{~g}: 0.055 \mathrm{~g}, 0.070 \mathrm{~g}, 0.085 \mathrm{~g}, 0.10 \mathrm{~g}, 0.115 \mathrm{~g}, 0.125 \mathrm{~g})$. $\mathrm{MgO}$ nanoparticle concentrations are $1.67 \mathrm{wt} \%, 5.00 \mathrm{wt} \%$, 10.0 wt $\%, 15.0$ wt\%, 20.0 wt\%, 25.0 wt\%, 30.0 wt\% (A : B : MgO nanoparticles $=1.50 \mathrm{~g}: 1.50 \mathrm{~g}: 0.05 \mathrm{~g}, 0.15 \mathrm{~g}, 0.30 \mathrm{~g}, 0.45 \mathrm{~g}$,

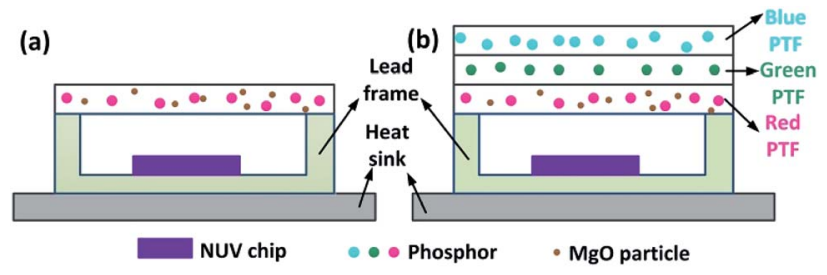

Fig. 1 Schematic diagram of PTFs: (a) red LED, (b) laminated white LED.

$0.60 \mathrm{~g}, 0.75 \mathrm{~g}, 0.90 \mathrm{~g})$. The specific concentration combination is shown in Fig. 2.

\section{Experiment process}

PTF preparation. The raw materials were weighed according to the preset concentrations, and the colloidal mixture was formed by a magnetic stirrer rotating at $200 \mathrm{rpm}$ for $20 \mathrm{~min}$, and then placed in a vacuum drying oven at a vacuum of $-0.1 \mathrm{MPa}$ for about $30 \mathrm{~min}$. During this process, the PTF forming molds were pre-heated and surface-sprayed with stripping agent, the temperature was $80^{\circ} \mathrm{C}$, the colloidal mixture was injected into the cavity, and the upper template was covered to maintain a temperature of $150{ }^{\circ} \mathrm{C}$ for $1.5 \mathrm{~h}$. Finally, different types of PTFs were obtained by cooling the mold.

Red LED and laminated white LED preparation. The chip was fixed in the SMD lead frame by solid crystal glue (Kyocera CT285 type), baked in an oven at $150{ }^{\circ} \mathrm{C}$ for $1 \mathrm{~h}$, and the electrode and lead frame were electrically interconnected by a wire bonding machine. Transparent silicone resin was injected into the cavity of the lead frame. PTFs were placed on the surface of the lead frame according to the structure of Fig. 1, and the layers of PTFs were bonded by the transparent silicone resin to eliminate the influence of air between the interfaces. After baking at $150{ }^{\circ} \mathrm{C}$ for $1 \mathrm{~h}$, the red LEDs and laminated white LEDs were obtained.

\section{Test analysis}

The microscopic morphology of PTF was observed by a Zeiss Evo18 scanning electron microscope at a voltage of $20 \mathrm{kV}$ and a magnification of $1000 \times$. The crystallinity was determined by a Rigaku Ultima IV X-ray diffractometer with a voltage of $40 \mathrm{kV}$, a scanning range of $10-90^{\circ}$, speed of $10^{\circ} \mathrm{min}^{-1}$, and the step length was $0.01^{\circ}$. The fluorescence lifetime was measured by an Edinburgh FLS980 steady state/transient fluorescence spectrometer. The reflectivity and transmittance and PCEs were

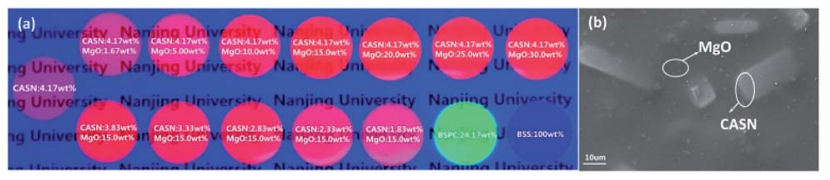

Fig. 2 (a) PTFs with different CSAN and MgO nanoparticle concentrations. (b) Micrograph of PTF with CASN (4.17 wt\%) and MgO nanoparticles (15.0 wt\%). 
tested and calculated by a Mu-Optical Double Integrating Sphere. The single-ball diameter is $30 \mathrm{~cm}$, and the inner wall is sprayed with $\mathrm{BaSO}_{4}$ powder to provide diffuse reflective conditions. PTF scattering distribution was determined using a light distribution tester built by our group, the components used including $10 \mathrm{~cm}$ diameter integrating sphere, rotating table, laser (410 nm, $660 \mathrm{~nm}$ ), etc. Red LED and laminated white LED light color performance was tested with an Everfine PMS-80 fluorescence spectrum analysis system with a range of 380$800 \mathrm{~nm}$, and the above tests were carried out at room temperature.

\section{Results and discussion}

Fig. 2(a) shows the combinations of different concentrations of CSAN and MgO nanoparticles in red PTFs and a mass fraction of 24.17 wt\% green PTF, $100 \mathrm{wt} \%$ of blue PTF under NUV light illumination. From Fig. 2(a) it can be seen that as the $\mathrm{MgO}$ nanoparticle or phosphor concentration increases, the transmittance of the PTFs gradually decreases, and the color exhibited is also different. Fig. 2(b) shows a micrograph of PTF prepared with CASN (4.17 wt\%) and $\mathrm{MgO}$ nanoparticles (15.0 wt\%). The image shows that phosphor and MgO nanoparticles are evenly distributed in silicone resin, the interfaces between particles and silicone resin are continuous, and no fault phenomenon occurs which is beneficial to photon propagation.

Fig. 3 shows XRD patterns of red PTFs of different CSAN and $\mathrm{MgO}$ nanoparticle concentrations. The $\mathrm{MgO}$ nanoparticle crystal form used belongs to periclase, corresponding to a cubic system, and the space group is $F m \overline{3} m$. The pattern is matched with standard card PDF\#77-2179. It can be seen from the figure that as the concentration increases, the positions of the diffraction peaks for the CASN and $\mathrm{MgO}$ nanoparticles remain unchanged, indicating that the crystal structures have not changed during the incorporation of $\mathrm{MgO}$ nanoparticles and the process of PTF formation.

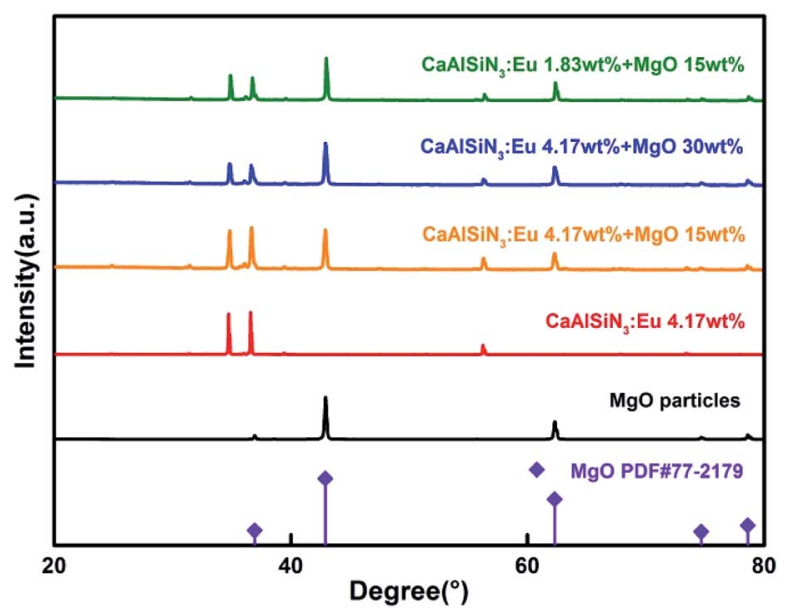

Fig. 3 XRD patterns of PTFs with different CSAN and MgO nanoparticle concentrations.
Fig. 4 shows the optical parameters of PTFs obtained by double-integral sphere. The lasers used are a $410 \mathrm{~nm}$ NUV laser and a $660 \mathrm{~nm}$ red laser. $410 \mathrm{~nm}$ is close to the NUV LED chip peak $(415 \mathrm{~nm})$ to measure the reflectance and transmittance of phosphor emission light. For the PCE of PTFs, the specific formula is as in eqn (1). ${ }^{37} 660 \mathrm{~nm}$ is used to measure the optical properties of PTFs for long-wavelength red light outside the excitation spectrum. It can be seen from Fig. 4(a) that as the $\mathrm{MgO}$ nanoparticle concentration increases, the reflectance at $660 \mathrm{~nm}$ increases and the transmittances decrease. Since the CASN excitation spectrum has no absorption effect at $660 \mathrm{~nm}$, the decrease is related to the scattering loss of the PTF. In Fig. 4(b) and (c), as the $\mathrm{MgO}$ nanoparticle concentration increases, the reflectance at $410 \mathrm{~nm}$ increases, the intensities of the reflected phosphor emission light increase first and then decrease, the transmittances at $410 \mathrm{~nm}$ decrease, and the transmitted light of phosphor gradually increases. In saturation, the PCEs in Fig. 4(d) first rise and then fall, which has the highest value of $83.15 \%$ at a concentration of $15.0 \mathrm{wt} \%$. The relevant change mechanism can be explained by Fig. 8 .

$$
\eta_{\mathrm{PCE}}=\frac{P_{\mathrm{RED}-\text { reflectance }}+P_{\mathrm{RED}-\text { transmittance }}}{P_{\text {Total }}-P_{\mathrm{NUV} \text {-reflectance }}-P_{\mathrm{NUV} \text {-transmittance }}}
$$

where $P_{\text {Total }}$ is the total optical power collected by two integrating spheres before adding of PTFs, $P_{\text {RED-transmittance }}$ and $P_{\text {NUV-transmittance }}$ are the optical power collected by integrating sphere away from the excitation source, while $P_{\text {RED-reflectance }}$ and $P_{\text {NUV-reflectance }}$ are the optical power collected by integrating sphere near the excitation source.

Fig. 5(a-c) shows light scattering distribution of $410 \mathrm{~nm} \mathrm{LD}$, phosphor emission light at $627 \mathrm{~nm}$ and $660 \mathrm{~nm}$ LD by PTFs with different CSAN and MgO nanoparticle concentrations. Fig. 5(d) shows the scattering distribution characteristics of CASN and $\mathrm{MgO}$ nanoparticles to photons of $410 \mathrm{~nm}, 627 \mathrm{~nm}$ and $660 \mathrm{~nm}$ calculated based on Mie theory. Fig. 5(a) and (c) have similar trends, in the concentration range of $0.00 \mathrm{wt} \%$ to $10.0 \mathrm{wt} \%$, and there is a peak in the range of $\pm 10^{\circ}$ of the scattered light intensity, that is, the main forward transmission is dominant at

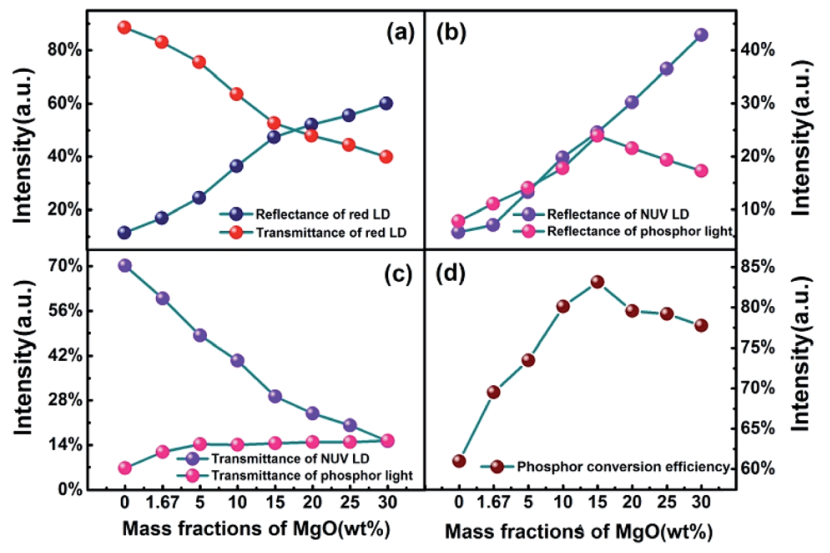

Fig. 4 Reflectance and transmittance of (a) $410 \mathrm{~nm}$, (b) $627 \mathrm{~nm}$, (c) $660 \mathrm{~nm}$ and (d) PCE of PTFs with different CSAN and MgO nanoparticle concentrations. 

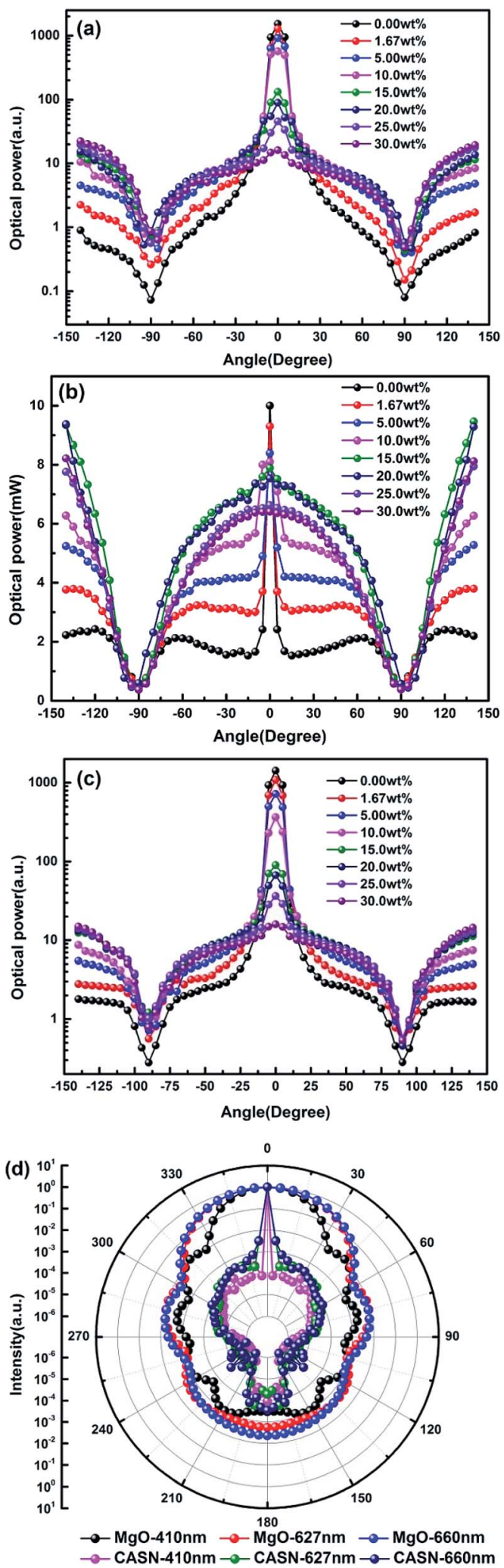

Fig. 5 Light scattering distribution of (a) $410 \mathrm{~nm}$, (b) $627 \mathrm{~nm}$ and (c) $660 \mathrm{~nm}$ by PTFs with different CSAN and MgO nanoparticle concentrations. (d) The scattering distribution characteristics of CASN and $\mathrm{MgO}$ nanoparticles to photons of $410 \mathrm{~nm}, 627 \mathrm{~nm}$ and $660 \mathrm{~nm}$ calculated based on Mie theory. low concentration, but as the concentration increases, the scattered light intensity gradually becomes smooth, which is attributed to the scattering effect of the $\mathrm{MgO}$ nanoparticles. The intensity gradually increases in the range of $90-140^{\circ}$ backscattering, which corresponds to the result of Fig. 4. Further, in combination with Fig. 5(d), it can be seen that the CASN also has scattering peaks in a small angle range. The phenomenon may be due to the particle size of up to $18.7 \mu \mathrm{m}$, for the propagating photons, which is similar to the lens. The light is concentrated in the range of the forward small angle. Due to the small particle size, the $\mathrm{MgO}$ nanoparticles have a diffraction effect on the photon propagation process, and strengths of forward and backward scattering are close. Fig. 5(b) is a light distribution diagram of phosphor emission light. There is also a peak phenomenon in the range of $\pm 10^{\circ}$, the peak gradually disappears as the concentration increases, and before and after the critical angle of $\pm 90^{\circ}$, the forward and backward intensities are close to each other, which indicates that the luminescence of phosphor is isotropic. The phosphor emission light intensity reaches the highest value at a concentration of $15 \mathrm{wt} \%$.

Fig. 6 shows the fluorescence lifetimes of PTFs at $\mathrm{MgO}$ nanoparticle concentrations of $0.00 \mathrm{wt} \%, 15.0 \mathrm{wt} \%$, and $30.0 \mathrm{wt} \%$. After a certain substance is excited and then fluoresces, and then the excitation light is removed, the fluorescence lifetime is the time for the fluorescence intensity to gradually decrease to $1 / e$ of the maximum intensity fluorescence $I_{0}$. The time can be used as a reference for the existence of energy transfer phenomena inside the material. According to the test results, a single exponential function fitting is performed to obtain the corresponding fluorescence lifetimes which are $629.68 \mathrm{~ns}, 617.11 \mathrm{~ns}$ and $615.25 \mathrm{~ns}$, respectively. It can be seen that as the concentration increases, the fluorescence lifetime decreases, which may be due to the scattering effect increased by $\mathrm{MgO}$ nanoparticle concentration. The probability of $\mathrm{Eu}^{2+}$ being excited increases, owing to an overlapping and self-absorption effect of excitation and emission spectra, and the energy transfer occurs in the luminescence center $\mathrm{Eu}^{2+}$ $\mathrm{Eu}^{2+}$, resulting in a shorter fluorescence lifetime. ${ }^{38,39}$

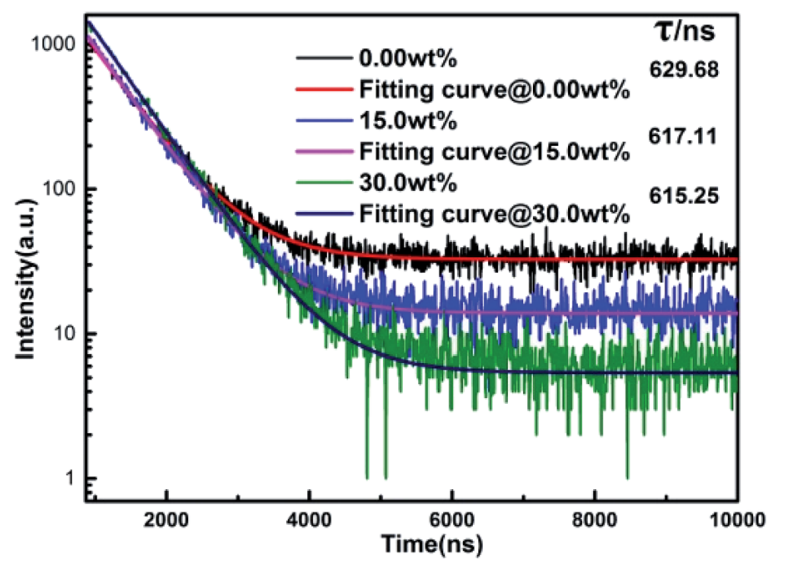

Fig. 6 Fluorescence lifetimes of PTFs at MgO nanoparticle concentrations of $0.00 \mathrm{wt} \%, 15.0 \mathrm{wt} \%$, and $30.0 \mathrm{wt} \%$. 
Fig. 7 is a graph showing the corresponding luminous flux and red/NUV ratios of red LEDs prepared according to Fig. 1 at different $\mathrm{MgO}$ nanoparticle concentrations. As can be seen from the figure, the overall variation of luminous flux is similar to that of Fig. 4(d), and there is a maximum value of $20.337 \mathrm{~lm}$ at $15.0 \mathrm{wt} \%$. The red/NUV ratio maintains an upward tendency, which indicates that the red emission intensity is always greater than that of the NUV light.

The regularity of PTFs and red LEDs and mechanism in Fig. 4-7 can be explained by the schematic diagram of photon propagation in the PTFs shown in Fig. 8. The regions A, B, and C indicate the PTFs without $\mathrm{MgO}$ nanoparticles (0.00 $\mathrm{wt} \%$ ), and with concentrations of (1.67 wt $\%, 5.00 \mathrm{wt} \%, 10.0 \mathrm{wt} \%$, $15.0 \mathrm{wt} \%)$ and $(20.0 \mathrm{wt} \%, 25.0 \mathrm{wt} \%, 30.0 \mathrm{wt} \%)$ respectively. In region $\mathrm{A}$, when $\mathrm{MgO}$ nanoparticles are not incorporated, NUV (Ray (1) can easily penetrate the PTF due to the low phosphor concentration, and the propagation mode is mainly the forward transmission, and the emission of the phosphor (Rays (3), (4)) spreads in an isotropic manner. In region $\mathrm{B}$, when $\mathrm{MgO}$ nanoparticles are doped, the direction of light propagation changes, and the absorption of NUV light and the forward transmission red emission light intensity increase significantly, mainly because the scattering effect of $\mathrm{MgO}$ nanoparticles improves the absorption probability of NUV light by the phosphor (Rays (8), (11) and red light forward transmission (Ray (10). The increase in the probability of NUV light retroreflection (Rays (6, (7)) is due to the increase refractive index of PTFs, ${ }^{33,40}$ as shown by the formula (2)-(5), enhancing the probability of total reflection of NUV light at the air interface, as shown by the formula (6) and (7), so the overall performance is that the retroreflective intensity of NUV light is enhanced, and forward transmission intensity is reduced.

$$
n_{\mathrm{PTF}}=\chi_{\mathrm{Phopshor}} n_{\mathrm{Phopshor}}+\chi_{\mathrm{MgO}} n_{\mathrm{MgO}}+\chi_{\mathrm{Silicon}} n_{\text {Silicon }}
$$

wherein $n_{\mathrm{PTF}}, n_{\mathrm{Phopshor}}, n_{\mathrm{MgO}}$ and $n_{\text {Silicon }}$ represent the refractive index of PTFs, red phosphor, MgO nanoparticles, and silicone resin, respectively, and $\chi_{\mathrm{Phopshor}}, \chi_{\mathrm{TiO}_{2}}$ and $\chi_{\text {Silicon }}$ represent the volume ratios of red phosphor, $\mathrm{MgO}$ nanoparticles, and silicone

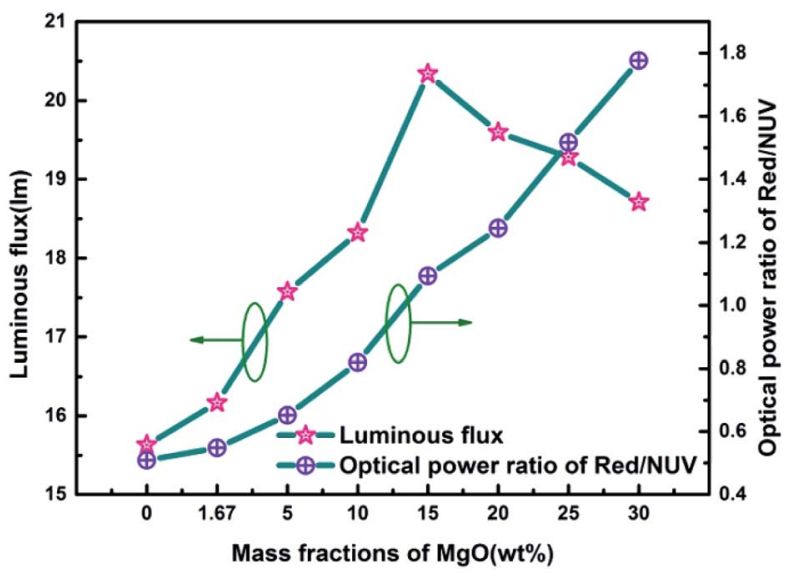

Fig. 7 Luminous efficiency and red/NUV ratios of red LEDs prepared using PTFs with different $\mathrm{MgO}$ nanoparticle concentrations.

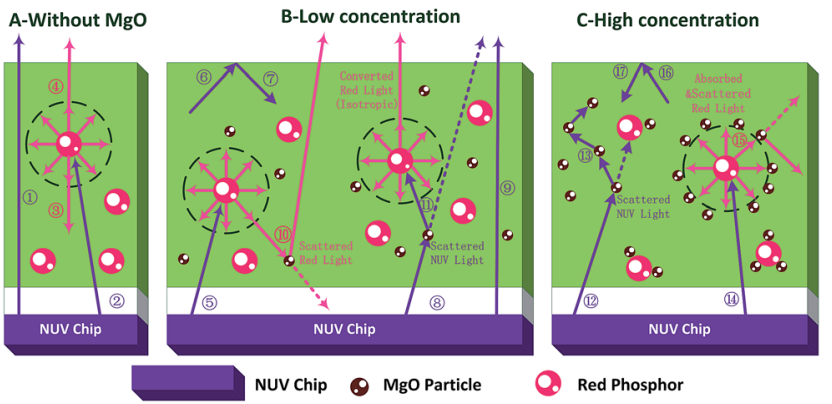

Fig. 8 Schematic diagram of light propagation direction in PTF at different $\mathrm{MgO}$ nanoparticle concentrations.

resin in the PTFs, $\chi_{\text {Phopshor }} n_{\text {Phopshor }}+\chi_{\text {Silicon }} n_{\text {Silicon }}$, which are constant according to known conditions. This is represented by the letter $C$, so the formula (2) can be expressed as:

$$
n_{\mathrm{PTF}}=C+\chi_{\mathrm{MgO}} n_{\mathrm{MgO}}
$$

The relationship of $\chi_{\mathrm{MgO}}$ with the $\mathrm{MgO}$ nanoparticle concentration $c_{\mathrm{MgO}}$ satisfies the following:

$$
c_{\mathrm{MgO}}=\chi_{\mathrm{MgO}} \rho_{\mathrm{MgO}}=\frac{4}{3} \pi r^{3} N(r)_{\mathrm{MgO}} \rho_{\mathrm{MgO}}
$$

In the formula, $N(r)_{\mathrm{MgO}}$ and $\rho_{\mathrm{MgO}}$ represent the concentration distribution function and the density of $\mathrm{MgO}$ nanoparticles which are constants, and the simultaneous eqn (3) and (4) are obtained:

$$
n_{\mathrm{PTF}}=C+\frac{c}{\rho_{\mathrm{MgO}}} n_{\mathrm{MgO}}
$$

It can be seen that the refractive index of the PTF is positively correlated with the $\mathrm{MgO}$ nanoparticle concentration.

The propagation of light at the interface between the PTF and the air satisfies the law of total reflection:

$$
n_{\mathrm{PTF}} \sin \alpha_{1}=n_{\mathrm{Air}} \sin \alpha_{2}
$$

when total reflection occurs, $\alpha_{2}=90^{\circ}$, and the critical angle $\alpha_{1}$ is:

$$
\alpha_{1}=\arcsin \frac{n_{\mathrm{Air}}}{n_{\mathrm{PTF}}}=\arcsin \frac{1}{C+\frac{c}{\rho_{\mathrm{MgO}}} n_{\mathrm{MgO}}}
$$

It is known that the refractive index of air is 1 , so the combination formula (7) shows that as the $\mathrm{MgO}$ nanoparticle concentration increases, the critical angle of total reflection decreases, and the probability of total reflection increases.

In region $C$, when the amount of $\mathrm{MgO}$ nanoparticles incorporated reaches a certain value, $\mathrm{MgO}$ nanoparticles will form an agglomeration on the phosphor. On the one hand, the $\mathrm{MgO}$ cluster will generate multiple scattering (Ray (13) to the excitation light, and on the other hand, the phosphor emission light 
(Ray (15) will fall into the $\mathrm{MgO}$ cluster, and multiple scattering direct energy depletion will occur, resulting in a decrease in the total optical power of the red emission light. At the same time, according to the formulas (5) and (7), the PTF refractive index continues to increase, the reflection phenomenon on the interface is enhanced, and the retroreflective intensity of NUV light is gradually increased.

Fig. 9 and 10 show corresponding CCTs, LEs, CRI and spectral distribution curves of different $\mathrm{MgO}$ nanoparticle concentrations for laminated white LEDs prepared according to Fig. 1(b). It can be seen that the CCTs and LEs decrease with increasing concentration, and the light source apparent color in the illustration changed gradually from positive white to red, indicating that the control of white LED color can be achieved by changing the $\mathrm{MgO}$ nanoparticle concentration. The CRI is greater than 90 for the mass fraction of $0 \mathrm{wt} \%$ to $10 \mathrm{wt} \%$, and its value becomes gradually lower than 90 with increasing $\mathrm{MgO}$ nanoparticle concentration and red phosphor emission light intensity. The spectral distribution curve in Fig. 10 can be used to explain this phenomenon. In the figure, the red light intensity first rises and then falls, with the largest value at $15.0 \mathrm{wt} \%$. The light source color changed gradually from positive white to red because the increasing red light intensity is higher than the blue and green light amplitude. The change of luminous efficiency can be explained by formula (8). In the range of visual efficiency curve of the visible spectrum, as the concentration increases, the green light intensity gradually decreases, although the red light intensity gradually increases, but away from the maximum peak of $555 \mathrm{~nm}$, so the luminous efficiency is gradually reduced according to the formula.

$$
\mathrm{LE}=K_{\mathrm{m}} \frac{\int V(\lambda) S(\lambda) \mathrm{d} \lambda}{I V} \operatorname{lm} \mathrm{W}^{-1}
$$

$V(\lambda)$ represents the visual efficiency curve for the visible spectrum; $S(\lambda)$ represents the spectral power density; $K_{\mathrm{m}}$ represents the spectral optical performance at a wavelength of $555 \mathrm{~nm}$, which is $683 \mathrm{~lm} \mathrm{~W}^{-1}$; and $I$ and $V$ represent the input current and voltage, respectively.

In order to more objectively evaluate the influence of $\mathrm{MgO}$ nanoparticles on laminated white LEDs, under similar color

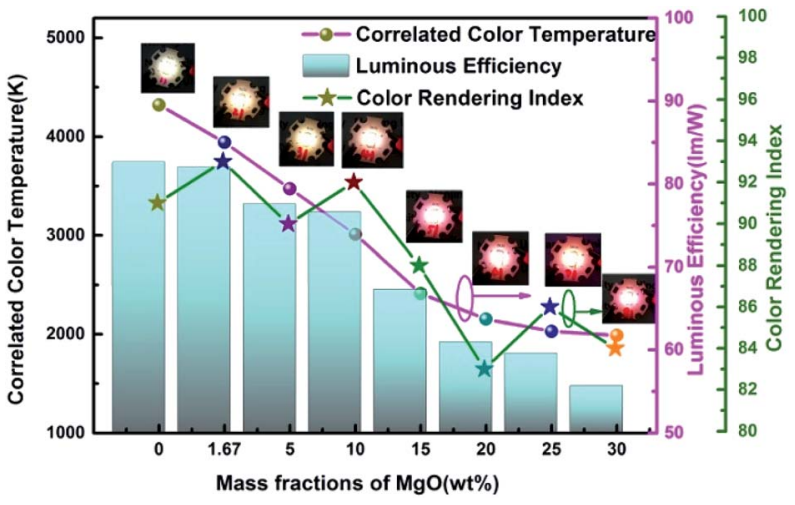

Fig. 9 Trends of CCT, LE and CRI of laminated white LEDs under different $\mathrm{MgO}$ nanoparticle concentrations.

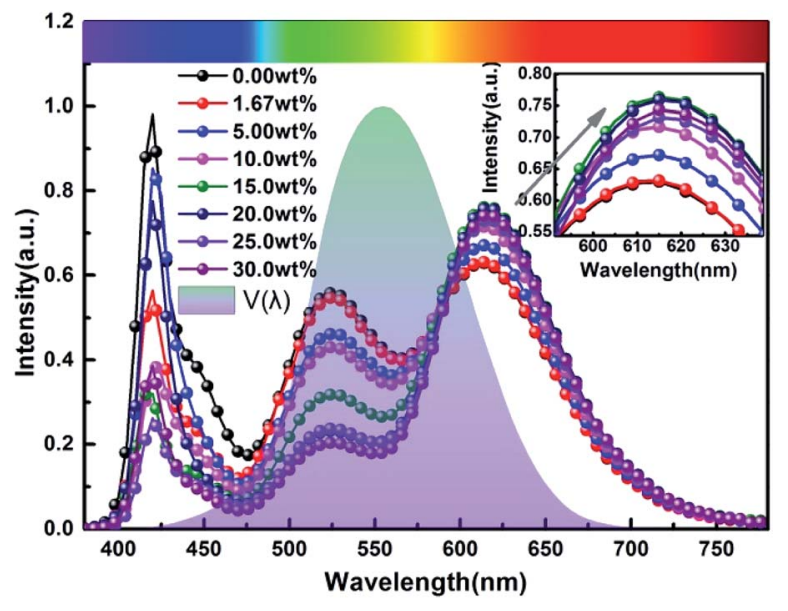

Fig. 10 Spectral distribution curves of laminated white LEDs under different $\mathrm{MgO}$ nanoparticle concentrations.

temperature conditions, we select an incorporated MgO nanoparticle concentration of $15 \mathrm{wt} \%$, and adjust the red phosphor concentration to achieve white LED performance regulation. According to the experiment, references 1, 2, and 3 in Fig. 11 respectively represent CCT, LER, and LE values of laminated white LED prepared by red phosphor (4.17 wt\%) without $\mathrm{MgO}$ nanoparticles incorporated. When the red phosphor concentration is $1.83 \mathrm{wt} \%$, the CCT of white LED is $4243 \mathrm{~K}$, which is 79 $\mathrm{K}$ different from the reference $4322 \mathrm{~K}$. The corresponding LER and LE are $296.03 \mathrm{~lm} \mathrm{~W}^{-1}$ and $73.72 \mathrm{~lm} \mathrm{~W}^{-1}$ respectively. In the figure, the three indicators gradually decrease with the increase of red phosphor. This phenomenon can also be explained by the spectral distribution curve of Fig. 12, and its trend is similar to that of Fig. 9. In Fig. 12, as the red phosphor concentration increases, the red luminescence intensity increases, the green luminescence intensity decreases, so the corresponding CCT and LE decrease, while the LER is higher than that of the reference light source before the concentration of $3.33 \mathrm{wt} \%$, which indicates that the relative green emission ratio that promotes the luminous flux before this concentration is

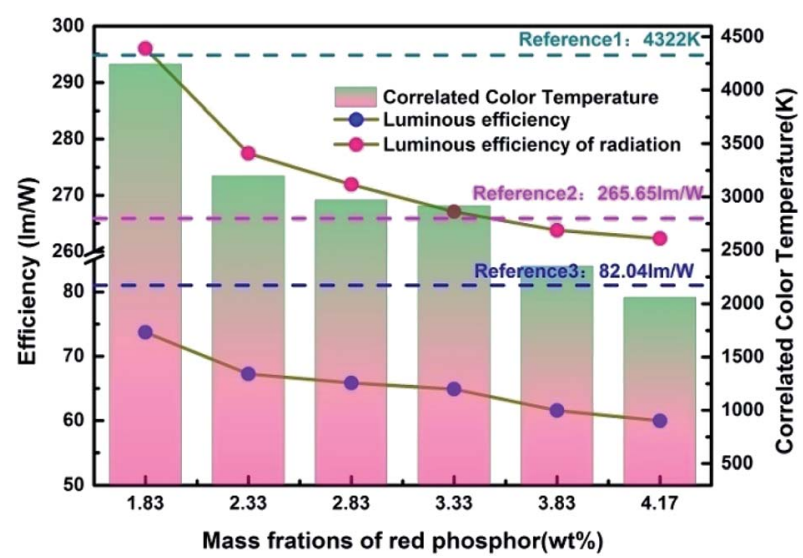

Fig. 11 CCTs and LEs of laminated white LEDs under different red phosphor concentrations. 


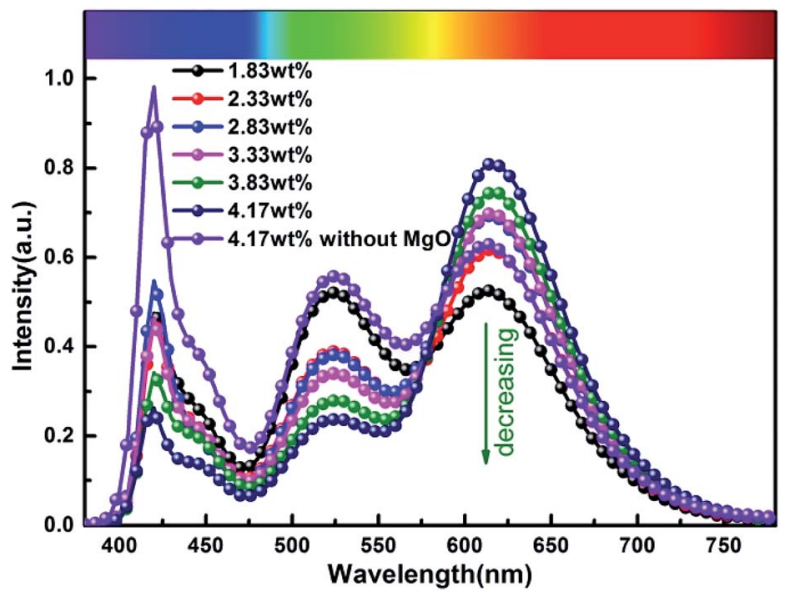

Fig. 12 Spectral distribution curves of laminated white LEDs under different red phosphor concentrations.

relatively high. After this concentration, since the red phosphor concentration is too high, NUV light is greatly absorbed and converted into red light, causing a decrease in the relative amount of NUV intensity for exciting green phosphor. It can be seen from the figure that under similar CCT conditions, the red phosphor amount is reduced from $4.17 \mathrm{wt} \%$ to $1.83 \mathrm{wt} \%$, and the saving amount reaches $56.12 \%$. At this time, LER and LE are $296.03 \mathrm{~lm} \mathrm{~W}^{-1}$ and $73.72 \mathrm{~lm} \mathrm{~W}^{-1}$ respectively. Compared with the reference value, the increase was $11.42 \%$, the decrease was $10.14 \%$, and the CRI increased from 90.6 to 91.8 .

$$
\mathrm{LER}=K_{\mathrm{m}} \frac{\int V(\lambda) S(\lambda) \mathrm{d} \lambda}{\int S(\lambda) \mathrm{d} \lambda} \operatorname{lm} \mathrm{W}^{-1}
$$

$V(\lambda)$ represents the visual efficiency curve for the visible spectrum; $S(\lambda)$ represents the spectral power density; $K_{\mathrm{m}}$ represents the spectral optical performance at a wavelength of $555 \mathrm{~nm}$, which is $683 \mathrm{~lm} \mathrm{~W}^{-1}$.

Fig. 13 and 14 respectively show the light distribution curve and CCT uniformity of laminated white LEDs corresponding to $\mathrm{MgO}$ nanoparticle concentration of $0.00 \mathrm{wt} \%$ (none incorporated) and $15.0 \mathrm{wt} \%$ (incorporated). It can be seen from Fig. 13 that the distribution of the two light sources is still of

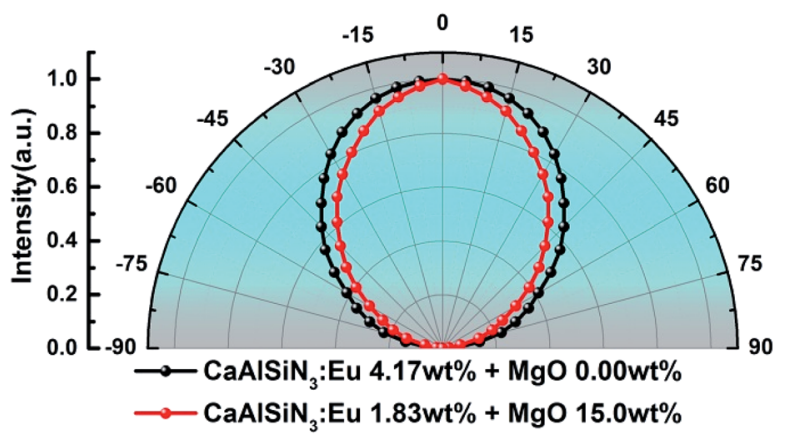

Fig. 13 Light distribution curve of laminated white LEDs corresponding to $\mathrm{MgO}$ nanoparticle concentration of $0.00 \mathrm{wt} \%$ (none incorporated) and $15.0 \mathrm{wt} \%$ (incorporated).

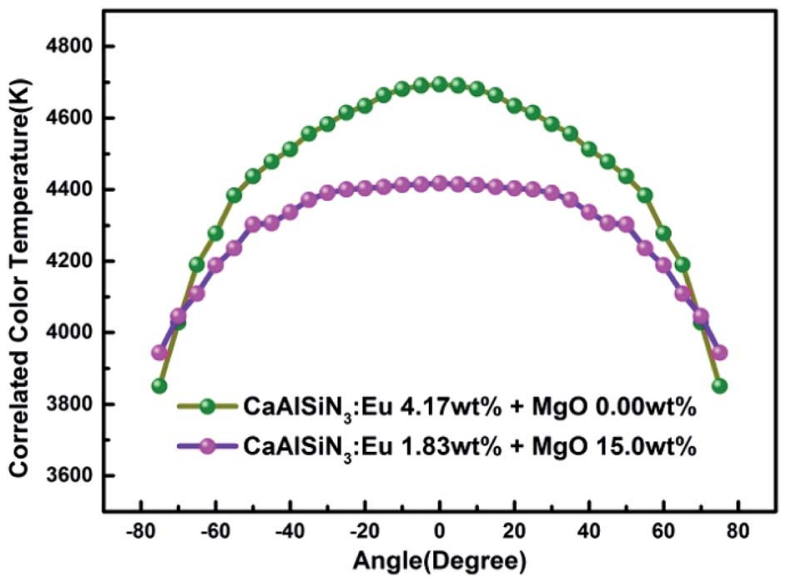

Fig. 14 CCT uniformity of laminated white LEDs corresponding to $\mathrm{MgO}$ nanoparticle concentration of $0.00 \mathrm{wt} \%$ (none incorporated) and 15.0 wt\% (incorporated).

Lambertian type (that is, the luminous intensity has a cosine distribution as a function of angle). In Fig. 14, in the range of $-75^{\circ}$ to $75^{\circ}$, the CCT range of none incorporated is $843 \mathrm{~K}$, the CCT uniformity is $82.04 \%$; the CCT range of doped type is $474 \mathrm{~K}$, the CCT uniformity is $89.27 \%$, showing $8.81 \%$ increases.

\section{Conclusions}

In this paper, red PTFs with different $\mathrm{MgO}$ nanoparticle concentrations were prepared based on a high-temperature molding process. The microscopic morphology and crystal structure of the PTFs were characterized by scanning electron microscopy and X-ray diffraction. Based on double integrating sphere and light color uniformity instrument measuring the reflection and transmittance, PCEs, spatial distribution of PTFs, and the fluorescence lifetimes under different concentration conditions were tested by steady state/transient fluorescence spectroscopy. The preparation of red LEDs and laminated white LEDs was further carried out. The above studies found that the $\mathrm{MgO}$ nanoparticles do not change the crystal structure of the material, and are dispersed uniformly in the silicone resin, based on its scattering effect. The PCEs of PTFs increase first and then decrease, reaching a maximum value of $83.15 \%$ at a concentration of $15 \mathrm{wt} \%$. The corresponding red LED has the highest luminous flux of $20.337 \mathrm{~lm}$. In the preparation of white LEDs via a lamination structure, it was found that the regulation of $\mathrm{MgO}$ nanoparticle concentration can effectively control the CCT of white LEDs from $4322 \mathrm{~K}$ to $1987 \mathrm{~K}$. Maintaining similar CCT and the used concentration of red phosphor of only $1.83 \mathrm{wt} \%$, the CCT of the prepared white LED is $4243 \mathrm{~K}$, which is different from the reference color temperature of $4322 \mathrm{~K}$ by 79 $\mathrm{K}$. The corresponding LER and LE are respectively $296.03 \mathrm{~lm}$ $\mathrm{W}^{-1}$ (increased by $11.42 \%$ ) and $73.72 \mathrm{~lm} \mathrm{~W}^{-1}$ (decreased by $10.14 \%$ ), the CRI increased from 90.6 to 91.8 , the phosphor usage was reduced by $56.12 \%$, and the CCT uniformity increased from $82.04 \%$ to $89.27 \%$, showing $8.81 \%$ increases. Studies have shown that the incorporation of $\mathrm{MgO}$ 
nanoparticles has an advantage for the improvement of red LED luminous flux. In the packaging application of laminated white LEDs, the amount of phosphors can be significantly reduced, the cost is reduced, and the CCT uniformity is improved, so that there is a potential application value in the preparation of highquality white LED packages.

\section{Conflicts of interest}

There are no conflicts to declare.

\section{Acknowledgements}

The authors would like to acknowledge the financial support in part from the National Key R\&D Program of China (grant no. 2016YFB0400600, 2016YFB0400605) and the Natural Science Foundation of Jiangsu Province (grant no. BK20171128).

\section{Notes and references}

1 P. Pust, P. J. Schmidt and W. Schnick, Nat. Mater., 2015, 14, 454-458.

2 H. O. Ji, J. Y. Su and R. D. Young, Light: Sci. Appl., 2014, 3, e141.

3 J. K. Sheu, S. J. Chang, C. H. Kuo, Y. C. Su, L. W. Wu, Y. C. Lin, W. C. Lai, J. M. Tsai, G. C. Chi and R. K. Wu, IEEE Photonics Technol. Lett., 2003, 15, 18-20.

4 S. H. Chuang, C. Y. Lin, S. l. Ou, C. S. Tsung, C. H. Chen and D. S. Wuu, Proc. SPIE, 2015, 9383, 93830R.

5 D. Cai, X. Yuan, D. H. Zhu and H. M. Zhou, Mater. Res. Bull., 2016, 85, 222-227.

6 S. P. Ying and H. Y. Chien, IEEE Trans. Electron Devices, 2016, 63, 1117-1121.

7 Y. M. Liu, J. Zou, M. M. Shi, Y. Li, B. B. Yang, Z. M. Wang, W. B. Li, F. Zheng, H. Y. Zhou and N. Jiang, J. Mater. Sci.: Mater. Electron., 2018, 29, 18476-18485.

8 Y. M. Liu, J. Zou, M. M. Shi, Y. Y. Li, B. B. Wang, Z. M. Li, W. B. Zheng, F. Zhou and H. Y. Jiang, J. Mater. Sci.: Mater. Electron., 2018, 29, 18476-18485.

9 Y. Peng, R. X. Li, H. Cheng, Z. Chen, H. Li and M. X. Chen, J. Alloys Compd., 2017, 693, 279-284.

10 S. Abe, J. Joos, L. I. D. J. Martin, H. Zeger and P. Smet, Light: Sci. Appl., 2017, 6, e16271.

11 S. W. Jeon, S. H. Kim, J. Choi, I. Jang, Y. Y. Song, W. H. Kim and J. P. Kim, Appl. Opt., 2018, 57, 5998-6003.

12 X. Shen, J. Mater. Sci.: Mater. Electron., 2017, 28, 1858518591.

13 Y. Liu, J. Zou, B. Yang, W. Li, M. Shi, Y. Han, Z. Wang, M. Li and N. Jiang, Mater. Technol., 2017, 33, 22-28.

14 X. L. Qiang, C. R. Zheng, M. M. Shi, B. B. Yang, Y. Li, Z. Z. Liu, F. Zheng and J. Zou, J. Mater. Sci.: Mater. Electron., 2016, 27, 13199-13208.

15 H. L. Kang, S. Kim, J. H. Oh, H. C. Yoon, J. H. Jo, H. Yang and Y. R. Do, Adv. Opt. Mater., 2018, 61, 1701239.
16 H. J. Kim, M. H. Shin, J. Y. Lee, J. H. Kim and Y. J. Kim, Opt. Express, 2017, 25, 10724-10734.

17 C. W. Kim, T. Y. Eom, I. S. Yang, B. S. Kim, W. I. Lee, Y. S. Kang and Y. S. Kang, Sci. Rep., 2017, 7, 1-9.

18 W. J. Ho, G. C. Yang, Y. T. Shen and Y. J. Deng, Appl. Surf. Sci., 2016, 365, 120-124.

19 C. N. Li, H. B. Rao, W. Zhang, C. Y. Zhou, Q. Zhang and K. Zhang, J. Disp. Technol., 2016, 12, 946-950.

20 X. J. Yu, B. Xie, Q. Chen, Y. P. Ma, R. K. Wu and X. B. Luo, IEEE Trans. Compon., Packag., Manuf. Technol., 2015, 5, 1253-1257.

21 Y. M. Liu, J. Zou, M. M. Shi, B. B. Yang, Y. Han, W. B. Li, Z. M. Wang, H. Y. Zhou, M. T. Li and N. Jiang, Ceram. Int., 2018, 44, 1091-1098.

22 Z. Z. Liu, B. B. Yang, C. Y. Zhang, Y. Li, J. Zou, M. M. Shi, X. L. Qian and F. Zheng, J. Lumin., 2019, 116652.

23 Z. Z. Liu, B. B. Yang, J. Zou, C. Y. Zhang, M. M. Shi, Y. Li, M. T. Li, Y. M. Liu, Z. M. Wang, F. Zhang and X. L. Qian, Opt. Mater., 2018, 86, 155-164.

24 O. H. Kwon, J. W. Jang, S. J. Park, J. S. Kim, S. J. Hong, Y. S. Jung, H. Yang, Y. J. Kim and Y. S. Cho, ACS Appl. Mater. Interfaces, 2019, 11, 1004-1012.

25 X. J. Xu, H. Li, Y. Zhuo, D. H. Xiong and M. X. Chen, J. Am. Ceram. Soc., 2019, 102, 1677-1685.

26 J. S. Kim, S. K. Eswaran, O. Kwon, S. J. Han, J. H. Lee and Y. S. Cho, Adv. Opt. Mater., 2016, 4, 1081-1087.

27 C. F. Lai, J. S. Li and C. W. Shen, ACS Appl. Mater. Interfaces, 2017, 9, 4851-4859.

28 H. Xiao, Y. J. Lu, Z. Q. Guo and Y. Lin, IEEE Photonics J., 2015, 7, 1-8.

29 O. Kwon, J. S. Kim, J. W. Jang and Y. S. Cho, Opt. Mater. Express, 2018, 8, 3230-3237.

30 J. S. Li, Z. T. Li, G. W. Liang and S. D. Yu, Opt. Express, 2016, 24, 23685-23696.

31 J. H. Oh, S. J. Yang and Y. R. Do, Opt. Express, 2013, 21, A765A773.

32 Y. Tang, Z. Li, Z. T. Li, J. S. Li, S. D. Yu and L. S. Rao, IEEE Trans. Electron Devices, 2017, 65, 158-163.

33 K. J. Chen, H. V. Han, H. C. Chen, C. C. Lin, S. H. Chien, C. C. Huang, T. M. Chen, M. H. Shin and H. C. Kuo, Nanoscale, 2014, 6, 5378-5383.

34 P. C. Wang, C. L. Lin, Y. K. Su, P. C. Chien, G. S. Huang, S. C. Kuo and G. C. Lyu, Thin Solid Films, 2014, 570, 273-276.

35 A. Q. D. Nguyen and V. H. Nguyen, J. Phys. Conf. Ser., 2017, 885, 012022.

36 P. Huang, H. Q. Shi, H. M. Xiao, Y. Q. Li, N. Hu and S. Y. Fu, Sci. Rep., 2017, 7, 5951.

37 N. Z. Zhuo, N. Zhang, B. C. Li, W. Q. Li, Q. Y. He, F. H. Shi, Y. H. Zhu, H. D. Xing and H. B. Wang, Acta Phys. Sin., 2016, 65, 058501.

38 S. Lee and K. S. Sohn, Opt. Lett., 2010, 35, 1004-1006.

39 S. X. Li, X. J. Liu, J. Q. Liu, H. L. Li, R. H. Mao, Z. R. Haung and R. J. Xie, J. Mater. Res., 2015, 30, 2919-2927.

40 W. Heller, J. Phys. Chem., 1965, 69, 1123-1129. 